caused by transverse aortic constriction (TAC) remains uncertain.

Methods For this study, 8 weeks old PMCA1Ht and wild type (WT) mice were grouped into TAC and sham operation cohorts. In mice subjected toTAC the thoracic aorta was ligated (over a curved blunt needle) between the brachiocephalic trunk and the left common carotid artery using a surgical suture that produces approximately 25\% narrowing of the aortic lumen, whilst in mice undergoing the sham operation the aorta was exposed and a surgical suture passed around it but not ligated. Echocardiogram and haemodynamic analysis were performed 5 week later and animals were sacrificed. Cardiac tissue samples were collected for histology, protein and RNA analysis.

Results At the basal level, PMCA1Ht and wild type mice had no significant difference in the cardiac structure and function on echocardiogram and haemodynamic analysis. Basal expression of PMCA1 was approximately 50\% lower in the PMCA1Ht mice whereas expression of other calcium handling genes including PMCA4, NCX, SERCA2 and RyR were similar in both groups. Five weeks after TAC, WT mice had worse LV function with a reduction in ejection fraction and fractional shortening, compared to PMCA1Ht mice from the same group. LV structure and function were preserved in the sham operation groups of both genotype. WT:TAC mice had a relatively higher expression of BNP, Collagen 1 and 3 compared to PMCA1Ht mice; however, expression of Bax (inducer of apoptosis) and $\mathrm{Bcl} 2$ (inhibitors of apoptosis) was similar in both genotypes. These findings suggest a higher degree of cardiac remodelling in the wild type mice as evidenced by worse LV function, higher expression of cardiac markers of hypertrophy and fibrosis. Interestingly in both the WT and PMCA1Ht groups post-TAC, there was a trend towards an increase in the mRNA expression of PMCA1 compared to the sham groups.

Conclusion Our data indicates that global heterozygous deletion of PMCA1 may have a protective role for the heart after TAC. It appears to reduce cardiac remodelling in a pressure overload setting. Authors recommend further work with larger sample size to understand molecular mechanisms underpinning these findings.

Conflict of Interest None

\section{A UNIQUE COMPARISON BETWEEN PRIMARY AND SECONDARY PULMONARY HYPERTENSION: 15-YEAR NATURALISTIC FOLLOW-UP STUDY FROM THE UNITED KINGDOM ACALM 'BIG DATA' REGISTRY}

${ }^{1}$ Rahul Potluri*, ${ }^{2}$ Debraj Das, ${ }^{3}$ Paul Carter, ${ }^{4}$ Billal Patel, ${ }^{4}$ Shajil Chalil, ${ }^{2}$ Wayne Tymchak, ${ }^{2}$ Daniel Kim, ${ }^{4}$ Ranjit More, ${ }^{2}$ Kevin Bainey. ${ }^{1}$ Blackpool Teaching Hospitals; ${ }^{2}$ Mazankowski Alberta Heart Institute; ${ }^{3}$ ACALM Study Unit; ${ }^{4}$ Blackpool Teaching Hospital NHS Foundation Trust

\subsection{6/heartjnl-2019-BCS.88}

Background Both primary and secondary pulmonary hypertension $(\mathrm{PH})$ are independently associated with morbidity and mortality. Compared to primary $\mathrm{PH}$, the underlying pathology in secondary PH remains diverse as seen in the WHO classification. As such, variation in long-term outcomes remains unclear. We examined the differences in clinical characteristics and long-term mortality between primary and secondary $\mathrm{PH}$ using the United Kingdom (UK) Algorithm for Co-morbidity, Associations, Length of stay and Mortality (ACALM) Registry. Methods Out of 1,816,230 patients admitted to several hospitals in Northern England from January 2000 to July 2014 (15-year follow-up), 2895 patients with PH were identified using the UK ACALM study protocol. ACALM utilizes a 'big data' approach to amalgamate multiple datasets and ICD-10/

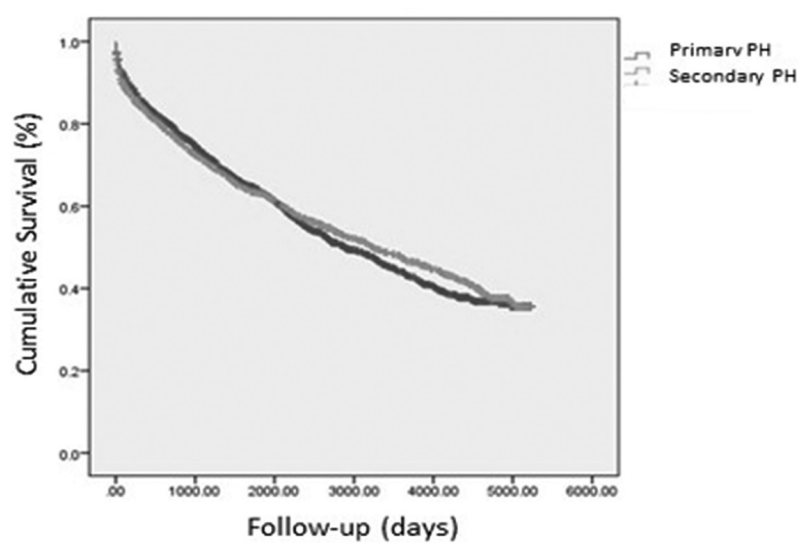

Abstract 90 Figure 1 Kaplan-Meier Survival Curves of Primary PH (Blue) and Secondary PH (Green) over 15-Years

Abstract 90 Table 1 Demographics, clinical characteristics and outcomes of patients with primary PH compared with secondary PH

\begin{tabular}{|c|c|c|c|}
\hline Co-Morbidity & Primary PH & Secondary PH & p value \\
\hline Age (Mean) & 63 & 66 & $p=0.110$ \\
\hline Female & $59.9 \%$ & $53.4 \%$ & $p=0.014$ \\
\hline Mean Charlson Score & 1.40 & 1.87 & $p<0.0001$ \\
\hline $\begin{array}{l}\text { Crude mortality } \% \\
1 \text { year } \\
5 \text { years } \\
10 \text { years }\end{array}$ & $\begin{array}{l}15.2 \\
36.7 \\
56.7\end{array}$ & $\begin{array}{l}16.7 \\
36.9 \\
53.3 \\
\end{array}$ & $p=0.018^{*}$ \\
\hline $\begin{array}{l}\text { Mean length of hospital } \\
\text { stay days (S.D) }\end{array}$ & $9.37(20.5)$ & $9.27(20.5)$ & $p=0.833$ \\
\hline $\begin{array}{l}\text { Mean number of } \\
\text { readmissions }(S . D)\end{array}$ & $7.93(37.5)$ & $7.41(8.5)$ & $P=0.193$ \\
\hline
\end{tabular}


OPCS-4 coding to identify patients with different diagnoses. In this study, primary $\mathrm{PH}$ was identified with an ICD-10 code of $\mathrm{I} 270$ (specific) and secondary $\mathrm{PH}$ included all other codes relating to $\mathrm{PH}$.

Results Of our $\mathrm{PH}$ cohort, 1688 patients had primary $\mathrm{PH}$ (58.3\%) and 1207 patients had secondary PH (41.7\%). Table 1 shows the differences in selected clinical characteristics between both groups as well as crude mortality rates, readmissions and length of hospital stay. Kaplan-Meier curves for long-term survival are shown in figure 1. Cox-regression was performed for survival and found lower adjusted mortality with secondary $\mathrm{PH}$ compared to primary $\mathrm{PH}$ (adjusted HR 0.87, 95\% CI [0.78-0.98], $\mathrm{p}=0.018$ ).

Conclusion Date from the UK-ACALM 'big data' registry confirms poor long-term prognosis with both primary and secondary $\mathrm{PH}$ over a 15-year follow-up. Surprisingly, one in three patients will have died by 5-years. Improved survival was observed in secondary $\mathrm{PH}$ compared to primary $\mathrm{PH}$ possibly reflecting the treatment of reversible causes of secondary $\mathrm{PH}$ Further studies are required to understand survival differences in this complex population, where therapies continue to evolve.

Conflict of Interest Nil

\section{THE RELATION BETWEEN THYROID DYSFUNCTION AND MORTALITY IN PATIENTS WITH CHRONIC HEART FAILURE}

${ }^{1}$ Nathan Samuel*, ${ }^{2}$ Joseph Cuthbert, ${ }^{2}$ Oliver Brown, ${ }^{2}$ Syed Kazmi, ${ }^{3}$ John GF Cleland, ${ }^{1}$ Alan Rigby, ${ }^{2}$ Andrew Clark. 'Hull York Medical School; ${ }^{2}$ Department of Academic Cardiology, Hull York Medical School; ${ }^{3}$ Robertson Centre for Biostatistics

\subsection{6/heartjnl-2019-BCS.89}

Introduction Thyroid dysfunction is common in patients with chronic heart failure (CHF), but there is conflicting

Abstract 91 Table 1 Demographics, clinical characteristics and pharmacological treatment of patients with heart failure according to thyroid status

\begin{tabular}{|c|c|c|c|c|c|}
\hline & M & $\begin{array}{c}\text { Hypothyroidism* } \\
\text { (TSH>4.70) }\end{array}$ & $\begin{array}{c}\text { Euthyroid } \\
\text { (TSH } 0.35-4.70 \text { ) }\end{array}$ & $\begin{array}{c}\text { Hyperthyroidism } \dagger \\
\text { (TSH<0.35) }\end{array}$ & P value \\
\hline $\mathrm{N}$ & & 312 & 4491 & 189 & \\
\hline Age (years) & 0 & $74 \pm 10$ & $73 \pm 11$ & $74 \pm 10$ & 0.14 \\
\hline Female (\%) & 0 & 41 & 37 & 58 & $<0.0001$ \\
\hline TSH (mU/l) & 0 & $6.40(5.40-8.50)$ & $1.70(1.20-2.50)$ & $0.15(0.09-0.27)$ & $N / A$ \\
\hline fT4 (pmol/l) & 0 & $13.0(11.0-15.0)$ & $N / A$ & $18.0(16.0-21.0)$ & $N / A$ \\
\hline $\begin{array}{l}\text { NT-proBNP } \\
(\mathrm{ng} / \mathrm{l})\end{array}$ & 357 & $1974(885-4296)$ & $1105(438-2545)$ & $1118(483-2860)$ & $<0.0001$ \\
\hline BMI $\left(\mathrm{kg} / \mathrm{m}^{2}\right)$ & 26 & $28.5 \pm 6.4$ & $28.8 \pm 6.1$ & $28.5 \pm 6.8$ & 0.51 \\
\hline Heart rate (bpm) & 88 & $77 \pm 18$ & $75 \pm 18$ & $77 \pm 16$ & 0.12 \\
\hline $\mathrm{AF}(\%)$ & 0 & 47 & 34 & 31 & $<0.0001$ \\
\hline $\mathrm{SBP}(\mathrm{mmHg})$ & 55 & $135 \pm 27$ & $140 \pm 26$ & $139 \pm 25$ & 0.0007 \\
\hline $\mathrm{DBP}(\mathrm{mmHg})$ & 55 & $78 \pm 15$ & $79 \pm 14$ & $75 \pm 13$ & 0.004 \\
\hline NYHA I (\%) & 21 & 19 & 22 & 20 & 0.38 \\
\hline II (\%) & & 41 & 48 & 47 & 0.06 \\
\hline III/IV (\%) & & 40 & 30 & 33 & 0.0008 \\
\hline HeFREF (\%) & 0 & 68 & 60 & 58 & 0.009 \\
\hline HeFNEF (\%) & & 32 & 40 & 42 & 0.009 \\
\hline IHD (\%) & 0 & 44 & 47 & 39 & 0.08 \\
\hline Hypertension (\%) & 0 & 37 & 41 & 45 & 0.29 \\
\hline $\mathrm{DM}(\%)$ & 0 & 22 & 24 & 27 & 0.39 \\
\hline COPD $(\%)$ & 0 & 8 & 9 & 10 & 0.82 \\
\hline Diuretic (\%) & 0 & 80 & 72 & 76 & 0.002 \\
\hline ACEi /ARB (\%) & 0 & 69 & 70 & 69 & 0.94 \\
\hline$\beta$-Blocker (\%) & 0 & 61 & 59 & 59 & 0.73 \\
\hline MRA (\%) & 0 & 31 & 20 & 24 & $<0.0001$ \\
\hline Statin (\%) & 0 & 45 & 51 & 48 & 0.07 \\
\hline Thyroxine (\%) & 0 & 22 & 6 & 48 & $<0.0001$ \\
\hline Amiodarone (\%) & 0 & 3 & 1 & 2 & 0.001 \\
\hline $\mathrm{Hb}(\mathrm{g} / \mathrm{dl})$ & 148 & $12.8 \pm 1.9$ & $13.3 \pm 1.8$ & $12.7 \pm 1.5$ & $<0.0001$ \\
\hline $\begin{array}{l}\text { Creatinine } \\
(\mu \mathrm{mol} / /)\end{array}$ & 83 & $110(88-145)$ & $99(81-124)$ & $95(78-129)$ & $<0.0001$ \\
\hline Urea $(\mathrm{mmol} / \mathrm{l})$ & 77 & $6.05(4.70-8.40)$ & $7.00(5.40-9.50)$ & $7.30(5.20-10.40)$ & $<0.0001$ \\
\hline $\mathrm{Na}^{+}(\mathrm{mmol} / \mathrm{l})$ & 77 & $137 \pm 3.9$ & $138 \pm 3.4$ & $138 \pm 4.0$ & $<0.0001$ \\
\hline $\mathrm{K}^{+}(\mathrm{mmol} / \mathrm{l})$ & 142 & $4.4 \pm 0.5$ & $4.4 \pm 0.5$ & $4.3 \pm 0.5$ & 0.36 \\
\hline
\end{tabular}

*Included 12 patients with overt hypothyroidism (TSH $>4.7 \mathrm{mU} / \mathrm{l}, \mathrm{fT} 4<7.8 \mathrm{pmol} / \mathrm{l})$, of whom 2 patients were on thyroxine replacement. tIncluded 43 patients with overt hyperthyroidism $(\mathrm{TSH}<0.35$, fT4 $>21.0 \mathrm{pmol} / \mathrm{l})$, of whom 5 patients were on thyroxine replacement.

$\mathrm{M}$, missing; TSH, thyroid stimulating hormone; $\mathrm{fT} 4$, serum free thyroxine level; N, number of patients; NT-proBNP, N-terminal prohormone of brain natriuretic peptide; BMI, body mass index; AF, Atrial Fibrillation; SBP, systolic blood pressure; DBP, diastolic blood pressure; NYHA, New York Heart Association classification; LVSD, left ventricular systolic dysfunction; IHD, ischaemic heart disease; DM, diabetes mellitus; COPD, chronic obstructive pulmonary disease; ACEi, angiotensin converting enzyme inhibitor; ARB, angiotensin II receptor blocker; ?7-Blocker, beta blocker; MRA, mineralocorticoid receptor antagonist; $\mathrm{Hb}$, haemoglobin; N/A, not applicable. 